\title{
Gaussian-state entanglement in a quantum beat laser
}

\author{
Rabia Tahira, ${ }^{1}$ Manzoor Ikram, ${ }^{1}$ Hyunchul Nha, ${ }^{2}$ and M. Suhail Zubairy ${ }^{1,3}$ \\ ${ }^{1}$ Centre for Quantum Physics, COMSATS Institute of Information Technology, Islamabad, Pakistan \\ ${ }^{2}$ Texas A\&M University at Qatar, Education City, P.O. Box 23874, Doha, Qatar \\ ${ }^{3}$ Institute of Quantum Studies and Department of Physics, Texas A\&M University, College Station, Texas 77843-4242, USA
}

(Received 25 September 2010; revised manuscript received 21 March 2011; published 23 May 2011)

\begin{abstract}
Recently quantum beat lasers have been considered as a source of entangled radiation [S. Qamar, F. Ghafoor, M. Hillery, and M. S. Zubairy, Phys. Rev. A 77, 062308 (2008)]. We investigate and quantify the entanglement of this system when the initial cavity modes are prepared in a Gaussian two-mode state, one being a nonclassical state and the other a thermal state. It is investigated how the output entanglement varies with the nonclassicality of the input Gaussian state, thermal noise, and the strength of the driving field.
\end{abstract}

DOI: 10.1103/PhysRevA.83.054304

PACS number(s): 03.67.Mn, 42.50.Dv, 03.65.Ud

Gaussian states play a central role in the continuousvariable $(\mathrm{CV})$ regime as they can be produced from reliable sources and controlled experimentally using an accessible set of operations due to, for example, beam splitters, phase shifters, and efficient homodyne detection. Entanglement between two Gaussian modes can be generated in the laboratory, like two output beams of a parametric down-converter [1]. A number of schemes for the generation of such kinds of entangled states for photons, atoms, and bright light sources have also been proposed [2-14].

A quantum beat laser is one of the examples of $\mathrm{CV}$ entangled systems where the gain medium is usually threelevel atoms in a $\mathrm{V}$ configuration interacting with two modes of radiation in a doubly resonant cavity [9-13]. In order to couple these two modes, the upper two levels of the atom are coherently driven by a strong classical field, making the two modes highly correlated [15-17]. Recently we considered the generation of two-mode-entangled states of cavity modes in a quantum beat laser [10], where the presence of entanglement between the two modes was demonstrated using Duan et al. [18], Simon [19], and Hillery and Zubairy [20] criteria for some specific initial states, such as squeezed vacuum, Fock, and coherent states. It was shown that entanglement can be maintained for a longer time with the increase in the strength of the driving field. In quantum beat lasers entanglement eventually vanishes and its characteristic time highly depends upon the initial states of the cavity field.

In another study we investigated the entanglement generation by beam splitter when a general Gaussian state is mixed with thermal noise on a lossless beam splitter [21]. In this report, we employ the same class of initial states in a quantum beat laser; that is, the two cavity modes are initially in a (nonclassical) Gaussian state mixed with thermal noise. In this case, we can systematically investigate the time evolution of entanglement due to the well-established Gaussian formalism and quantify the degree of entanglement using the logarithmic negativity [22], not only determining the presence of entanglement contrasting the previous work [10], in a quantum beat laser.

Our model for the quantum beat laser is the same as that discussed in [15,17], that is, a three-level atomic system of $\mathrm{V}$ configuration in a doubly resonant cavity, as shown in Fig. 1. The atoms are prepared in coherent superposition of $|a\rangle$ and $|b\rangle$ by an external driving field with Rabi frequency $\Omega$. The atomic decay rates are assumed to be the same to simplify analysis. The evolution of the two-mode field in a quantum beat laser can be described by the characteristic function

$$
\chi\left(\zeta_{1}, \zeta_{2}, t\right)=\operatorname{Tr}\left(\rho(t) e^{\zeta_{1} a_{1}^{\dagger}-\zeta_{1}^{*} a_{1}} e^{\zeta_{2} a_{2}^{\dagger}-\zeta_{2}^{*} a_{2}}\right),
$$

where $a_{i}\left(a_{i}^{\dagger}\right)$ is the annihilation (creation) operator of the $i$ th field mode $(i=1,2)$ and $\rho(t)$ is the density matrix of the two-mode field given by the master equation [10]

$$
\begin{aligned}
\dot{\rho}(t)= & -\frac{1}{2}\left\{\alpha_{11}^{*} a_{1} a_{1}^{\dagger} \rho+\alpha_{11} \rho a_{1} a_{1}^{\dagger}-\left(\alpha_{11}+\alpha_{11}^{*}\right) a_{1}^{\dagger} \rho a_{1}\right\} \\
& -\frac{1}{2}\left\{\alpha_{22}^{*} a_{2} a_{2}^{\dagger} \rho+\alpha_{22} \rho a_{2} a_{2}^{\dagger}-\left(\alpha_{22}+\alpha_{22}^{*}\right) a_{2}^{\dagger} \rho a_{2}\right\} \\
& -\frac{1}{2}\left\{\alpha_{21}^{*} a_{1}^{\dagger} a_{2} \rho+\alpha_{12} \rho a_{1}^{\dagger} a_{2}-\left(\alpha_{12}+\alpha_{21}^{*}\right) a_{1}^{\dagger} \rho a_{2}\right\} e^{i \Phi} \\
& -\frac{1}{2}\left\{\alpha_{12}^{*} a_{1} a_{2}^{\dagger} \rho+\alpha_{21} \rho a_{1} a_{2}^{\dagger}-\left(\alpha_{21}+\alpha_{12}^{*}\right) a_{2}^{\dagger} \rho a_{1}\right\} e^{-i \Phi} \\
& -k_{1}\left(a_{1}^{\dagger} a_{1} \rho-2 a_{1} \rho a_{1}^{\dagger}+\rho a_{1}^{\dagger} a_{1}\right) \\
& -k_{2}\left(a_{2}^{\dagger} a_{2} \rho-2 a_{2} \rho a_{2}^{\dagger}+\rho a_{2}^{\dagger} a_{2}\right),
\end{aligned}
$$

where $k_{1}$ and $k_{2}$ are the decay rates for the cavity modes of frequencies $v_{1}$ and $v_{2}$, respectively. The phase angle $\Phi$ in the above equation is given by $\Phi=\phi+\left(v_{1}-v_{2}-v_{3}\right) t$, where $\nu_{3}$ is the frequency and $\phi$ is the phase of the external driving field. With the detunings $\Delta_{1} \equiv \omega_{a}-\omega_{c}-v_{1}$ and $\Delta_{2} \equiv \omega_{b}-$ $\omega_{c}-\nu_{2}$ and the atomic decay rate $\gamma$, the coefficients $\alpha_{i j}$ are given by

$$
\begin{aligned}
\alpha_{1(2), 1}= & \frac{g^{2} r_{a}}{2 \gamma\left(\gamma^{2}+\Omega^{2}\right)}\left\{\frac{\left(2 \gamma^{2}+\Omega^{2}+i \gamma \Omega\right)[\gamma-i(\Delta-\Omega / 2)]}{\gamma^{2}+(\Delta-\Omega / 2)^{2}}\right. \\
\pm & \left.\frac{\left(2 \gamma^{2}+\Omega^{2}-i \gamma \Omega\right)[\gamma-i(\Delta+\Omega / 2)]}{\gamma^{2}+(\Delta+\Omega / 2)^{2}}\right\} \\
\alpha_{1(2), 2}= & \frac{g^{2} r_{a} \Omega}{2 \gamma\left(\gamma^{2}+\Omega^{2}\right)}\left\{\frac{\gamma-i(\Delta-\Omega / 2)}{\gamma^{2}+(\Delta-\Omega / 2)^{2}}(\Omega-i \gamma)\right. \\
& \left.\mp \frac{\gamma-i(\Delta+\Omega / 2)}{\gamma^{2}+(\Delta+\Omega / 2)^{2}}(\Omega+i \gamma)\right\}
\end{aligned}
$$

where we set $g_{1}=g_{2}=g$ and $\Delta_{1}=\Delta_{2}=\Delta$. The coefficients $\alpha_{11}$ and $\alpha_{22}$ characterize the gain of the cavity modes while $\alpha_{12}$ and $\alpha_{21}$ are related to the atomic coherence produced between the levels $|a\rangle$ and $|b\rangle$ by the classical driving field. 


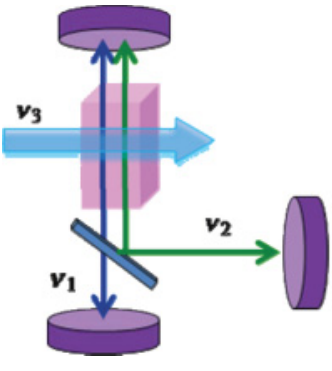

(a)

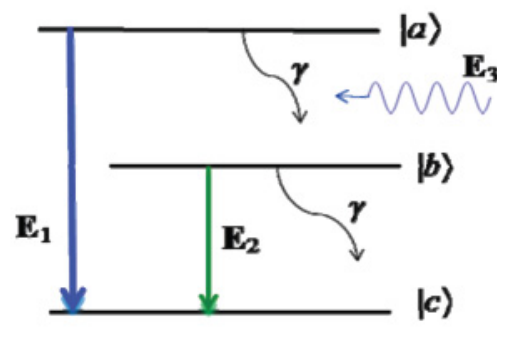

(b)
FIG. 1. (Color online) (a) Atomic medium inside a doubly resonant cavity. (b) Atomic level configuration.

When the output two-mode state in the quantum beat laser is Gaussian, its characteristic function is given by

$$
\chi\left(\zeta_{1}, \zeta_{2}, t\right)=\exp \left(-\frac{1}{2} \mathbf{y}^{\dagger} V_{t} \mathbf{y}\right),
$$

where $\mathbf{y}^{\dagger}=\left(\zeta_{1}^{*}, \zeta_{1}, \zeta_{2}^{*}, \zeta_{2}\right)$ and $V_{t}$ is the time-dependent covariance matrix of the form

$$
V_{t}=\left(\begin{array}{cc}
A & C \\
C^{\dagger} & B
\end{array}\right) .
$$

The submatrices $A, B$, and $C$ are given by

$$
\begin{gathered}
A=\left(\begin{array}{cc}
n_{1}+\frac{1}{2} & -m_{1} \\
-m_{1}^{*} & n_{1}+\frac{1}{2}
\end{array}\right), \quad B=\left(\begin{array}{cc}
n_{2}+\frac{1}{2} & -m_{2} \\
-m_{2}^{*} & n_{2}+\frac{1}{2}
\end{array}\right) \\
C=\left(\begin{array}{cc}
m_{s} & -m_{c} \\
-m_{c}^{*} & m_{s}^{*}
\end{array}\right)
\end{gathered}
$$

where $n_{1}=\left\langle a_{1}^{\dagger} a_{1}\right\rangle, \quad n_{2}=\left\langle a_{2}^{\dagger} a_{2}\right\rangle, \quad m_{1}=\left\langle a_{1}^{2}\right\rangle, \quad m_{2}=\left\langle a_{2}^{2}\right\rangle$, $m_{s}=\left\langle a_{1} a_{2}^{\dagger}\right\rangle$, and $m_{c}=\left\langle a_{1} a_{2}\right\rangle$ are time-dependant secondorder moments of the two-mode field and can be determined by the master equation (2).

We assume that the two modes of the cavity field are initially in a product state, which consists of two independent Gaussian states with one input in an arbitrary single-mode Gaussian state defined by nonclassicality $\tau$ and purity $u$ and the other input in a thermal state of average photon number $\bar{n}$. The covariance matrices for these two initial modes are given as [21]

$$
V_{1}=\left(\begin{array}{cc}
a & b \\
b^{*} & a
\end{array}\right), \quad V_{2}=\left(\begin{array}{cc}
\bar{n}+\frac{1}{2} & 0 \\
0 & \bar{n}+\frac{1}{2}
\end{array}\right)
$$

where matrix elements $a, b=|b| e^{i \theta}$, and $\bar{n}$ are given as

$$
\begin{gathered}
a=\frac{1}{4 u^{2}(1-2 \tau)}+\frac{1}{4}(1-2 \tau), \\
|b|=\frac{1}{4 u^{2}(1-2 \tau)}-\frac{1}{4}(1-2 \tau), \\
\bar{n}=\left(e^{\frac{h v}{k_{B} T}}-1\right)^{-1}
\end{gathered}
$$

where $k_{B}$ is the Boltzmann constant and $T$ is the absolute temperature of the thermal state. Thus, its characteristic function can be written as $\chi\left(\zeta_{1}, \zeta_{2}, t=0\right)=\exp \left(-\frac{1}{2} \mathbf{y}^{\dagger} V_{\text {in }} \mathbf{y}\right)$, where $V_{\text {in }}=V_{1} \oplus V_{2}$ is the initial covariance matrix. The characteristic function of the two-mode Gaussian state at time $t$ is given by Eq. (5), where the time-dependent elements of the covariance matrix $V_{t}$ at resonance $(\Delta=0)$ can be obtained as

$$
\begin{aligned}
n_{1}= & \frac{A_{11}}{D_{11}}+\frac{e^{-\alpha t}}{D_{11}}\left\{A_{12}-2\left[2 G-k\left(4+\chi^{2}\right)\right]\right. \\
& \left.\times\left[B_{11} \sin (\beta t)+B_{12} \cos (\beta t)\right]\right\}, \\
n_{2}= & \frac{A_{21}}{D_{22}}+\frac{e^{-\alpha t}}{D_{22}}\left\{A_{22}+2\left[2 G-k\left(4+\chi^{2}\right)\right]\right. \\
& \left.\times\left[B_{21} \sin (\beta t)+B_{22} \cos (\beta t)\right]\right\},
\end{aligned}
$$

$$
\begin{aligned}
m_{s}= & \frac{-i e^{-i \phi} \chi}{D_{11}}\left(A_{31}-e^{-\alpha t}\left\{A_{32}-\left[-2 G+k\left(4+\chi^{2}\right)\right]\right.\right. \\
& \left.\left.\times\left[B_{31} \sin (\beta t)+B_{32} \cos (\beta t)\right]\right\}\right),
\end{aligned}
$$

$$
\begin{aligned}
m_{1}= & \frac{1}{2\left(2+3 \chi^{2}+\chi^{4}\right)} b e^{i \theta} e^{-\alpha t}\left[\left(4+\chi^{2}\right) \chi^{2}\right. \\
& \left.+\left(4+2 \chi^{2}+\chi^{4}\right) \cos (\beta t)-2 Y \sin (\beta t)\right], \\
m_{2}= & -\frac{\left(4+\chi^{2}\right)^{2} \chi^{2}}{2 Y^{2}} b e^{i(\theta+2 \phi)} e^{-\alpha t}[1-\cos (\beta t)], \\
m_{c}= & i \frac{\left(4+\chi^{2}\right) \chi}{\left(2+3 \chi^{2}+\chi^{4}\right) Y} b e^{i(\theta+\phi)} e^{-\alpha t}\{Y[\cos (\beta t)-1] . \\
+ & \left.\left(2+3 \chi^{2}+\chi^{4}\right) \sin (\beta t)\right\},
\end{aligned}
$$

where $\chi=\Omega / \gamma, G=g^{2} r_{a} / \gamma^{2}$, and

$$
\alpha=2 k-\frac{4 G}{\left(4+\chi^{2}\right)}, \quad \beta=\frac{2 G Y}{4+5 \chi^{2}+\chi^{4}} .
$$

The other coefficients $A_{i j}, B_{i j}$, and $D_{i i}$ are given in the Appendix.

Entanglement among two modes of quantum beat laser can be determined using logarithmic negativity [22],

$$
N=\max \left\{0,-\log _{2}\left(2 \xi_{-}\right)\right\}+\max \left\{0,-\log _{2}\left(2 \xi_{+}\right)\right\},
$$

where $\xi_{ \pm}$are the two positive roots of the characteristic equation

$$
\xi^{4}-(\operatorname{Det}[A]+\operatorname{Det}[B]-2 \operatorname{Det}[C]) \xi^{2}+\operatorname{Det}\left[V_{\mathrm{t}}\right]=0 \text {. }
$$

Thus, entanglement depends upon the parameters $\Omega, \tau, u$, and $\bar{n}$. The major controlling parameter which greatly affects the entanglement is the strength of the driving field $\Omega$, which produces the coherence between the two lasing levels and is essentially responsible for entanglement. Here we discuss the two extreme cases of the driving field upon entanglement.

Case $(i)$ : Weak driving field $(\Omega \ll \gamma)$. When the strength of the driving field is much less than the spontaneous decay rates of the atomic levels at resonance $(\Delta=0)$ then the terms corresponding to the atomic coherence $\alpha_{12}$ and $\alpha_{21}$ are very small compared to the gain terms $\alpha_{11}$ and $\alpha_{22}$, resulting in no entanglement at all times.

Case (ii): Strong driving field $(\Omega \gg \gamma)$. It has been shown earlier that the lasing process in the quantum beat laser occurs only in the limit that the classical driving field is very strong [16]. With a very strong driving field $\Omega \gg \gamma$ at resonance $(\Delta=0)$, the coefficients corresponding to the gain $\alpha_{11}$ and $\alpha_{22}$ are much smaller than the coherence terms $\alpha_{12}$ and $\alpha_{21}$. So under this approximation the master equation describing the 
quantum beat laser reduces to

$$
\begin{aligned}
\dot{\rho}(t)= & -i\left[H_{\mathrm{eff}}, \rho\right]-k_{1}\left(a_{1}^{\dagger} a_{1} \rho-2 a_{1} \rho a_{1}^{\dagger}+\rho a_{1}^{\dagger} a_{1}\right) \\
& -k_{2}\left(a_{2}^{\dagger} a_{2} \rho-2 a_{2} \rho a_{2}^{\dagger}+\rho a_{2}^{\dagger} a_{2}\right),
\end{aligned}
$$

with the effective Hamiltonian

$$
H_{\mathrm{eff}}=\frac{2 i G \gamma}{\Omega}\left(a_{1}^{\dagger} a_{2} e^{i \Phi}+a_{1} a_{2}^{\dagger} e^{-i \Phi}\right) .
$$

Therefore, in the strongly driven limit, the two-mode quantum beat laser behaves like an optical beam splitter with some losses. It has already been shown that two output beams of a beam splitter can become entangled if one of the input beams is nonclassical [21,23-25].

For the general case, here we discuss the effect of various parameters $\Omega, \tau, u$, and $\bar{n}$ on entanglement. In Fig. 2(a) we
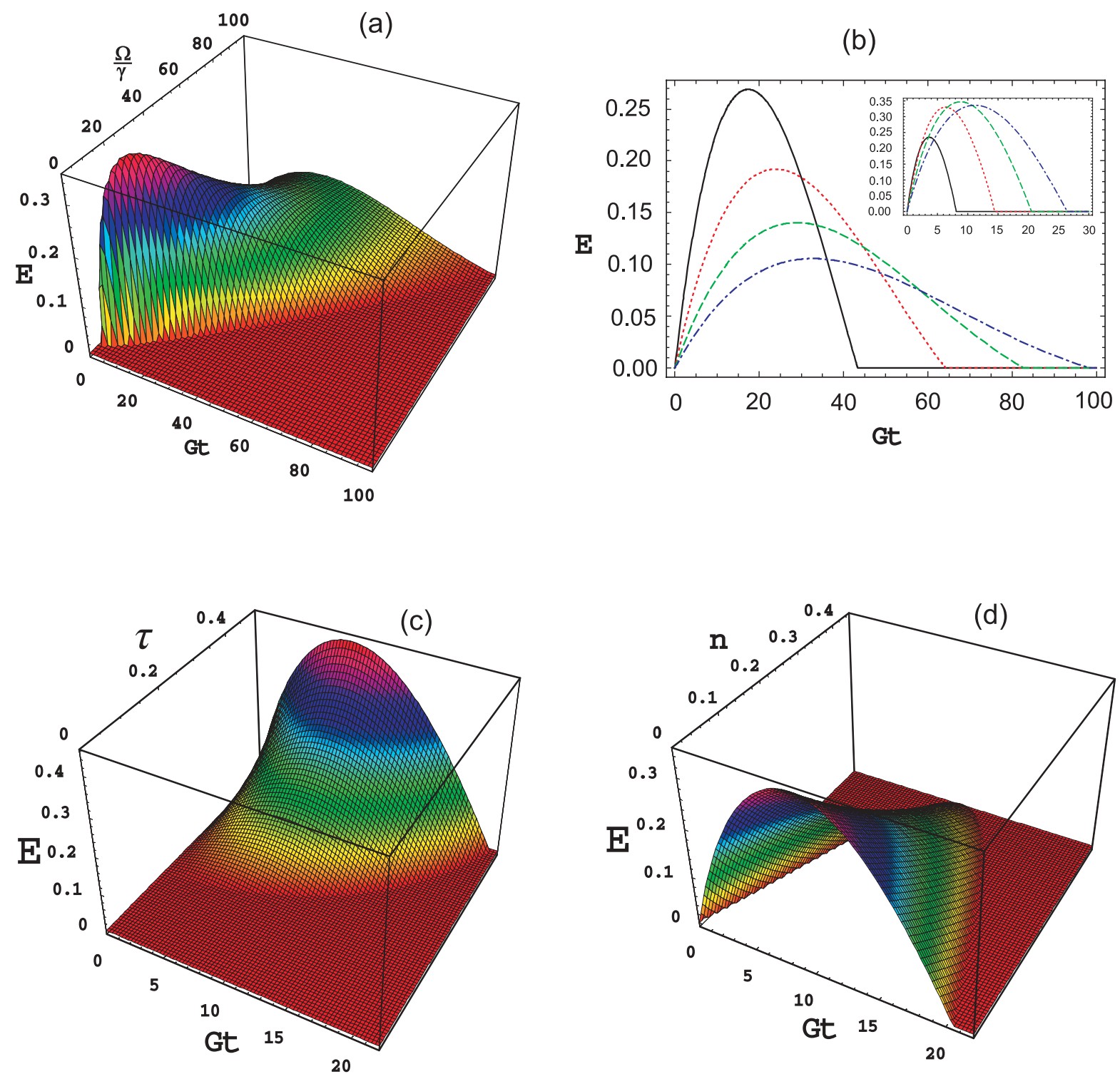

FIG. 2. (Color online) (a) Entanglement as a function of driving field strength $\Omega / \gamma$ and dimensionless time $G t$ at $\Delta=0, \bar{n}=0, u=1$, and $\tau=0.45$. (b) Cross-sections of Fig. 2(a) at, from top to bottom, $\Omega=800 \mathrm{kHz}$ (black solid), $1200 \mathrm{kHz}$ (red dotted), $1600 \mathrm{kHz}$ (green dashed), and $2000 \mathrm{kHz}$ (blue dash-dotted). Inset at smaller values of Rabbi frequency $\Omega=200 \mathrm{kHz}$ (black solid), $300 \mathrm{kHz}$ (red dotted), $400 \mathrm{kHz}$ (green dashed), and $500 \mathrm{kHz}$ (blue dash-dotted). (c) Entanglement as a function of nonclassicality $\tau$ and dimensionless time $G t$ at $\Delta=0, \bar{n}=0$, $u=1$, and $\Omega=400 \mathrm{kHz}$. (d) Entanglement as a function of average photon numbers $\bar{n}$ in the thermal state and dimensionless time $G t$ at $\Delta=0, \tau=0.45, u=1$ and $\Omega=400 \mathrm{kHz}$. The other parameters are taken as $\gamma=20 \mathrm{kHz}, r_{a}=22 \mathrm{kHz}, g=43 \mathrm{kHz}$, and $k=1.5 \mathrm{kHz}$.

show the effect of $\Omega$ on the entanglement. The value of $\Omega$ must be larger than $\sqrt{2} \gamma$ as the lesser values make $\beta$ in Eq. (18) complex, resulting in no entanglement. The amount of entanglement, which is very small at low $\Omega$, increases with $\Omega$ reaching the maximum value at about $\Omega \approx 20 \gamma$, and then begins to decrease with $\Omega$. The duration of the entanglement generally increases with the strength of the driving field $\Omega$, which is clearly demonstrated in Fig. 2(b) where the cross sections of the plot in Fig. 2(a) are shown. In Fig. 2(c) we show the effect of nonclassicality of the input Gaussian state upon entanglement. The amount of entanglement along with the survival time of the entanglement increases with the nonclassicality $\tau$ and approaches to its maximum value as $\tau \rightarrow 1 / 2$. In Fig. 2(d) we show the effect of thermal noise on entanglement. Increase of the thermal noise reduces the

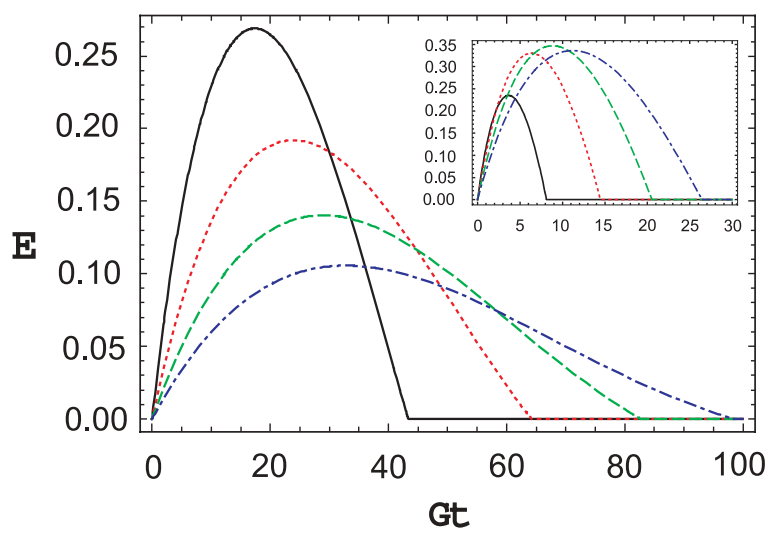

(b) 
amount and the duration of entanglement. The purity of the initial Gaussian state does not show any significant effect on entanglement.

This work is supported by NPRP Grant No. 1-7-7-6 from the Qatar National Research Fund (QNRF).

\section{APPENDIX: COEFFICIENTS OF THE MARTIX ELEMENTS}

The coefficients $A_{i j}, B_{i j}$, and $D_{i i}$ of the Eqs. (14)-(20) are.

$$
\begin{aligned}
A_{11}= & -8 G^{2} Y\left(1+3 \chi^{2}+\chi^{4}\right)\left(2 Z+k^{2}\left(14-2 \chi^{2}-\chi^{4}\right)\right), \\
A_{12}= & 2 G Y Z \chi^{2}[16 k+8(G-2 k)(2 a-\bar{n})+2(2 k(2-\bar{n}-4 a) \\
& \left.+G(3+2 a+2 \bar{n})) \chi^{2}+k(1-2 a-2 \bar{n}) \chi^{4}\right], \\
A_{21}= & -8 G^{2} Y^{3} \chi^{2}\left[2 G^{2}-6 G k+3 k^{2}\left(4+\chi^{2}\right)\right], \\
A_{22}= & 2 G Y Z \chi^{2}\left(4+\chi^{2}\right)\left[2 G\left((3+2 \bar{n}) \chi^{2}-4 \bar{n}\right)\right. \\
& -k\left(\left(2 n\left(\chi^{2}-2\right)-\chi^{2}-4\right)+2 a\left(2 G-k\left(4+\chi^{2}\right)\right)\right) \\
& \left.\times\left(4+\chi^{2}\right)\right], \\
A_{31}= & 8 G^{2} Y k(G-3 k)\left(8+14 \chi^{2}+7 \chi^{4}+\chi^{6}\right), \\
A_{32}= & 2 G Y Z[16 k+8(G-2 k)(2 a-\bar{n})+2(2 k(2-4 a-n) \\
& \left.+G(3+2 a+2 \bar{n})) \chi^{2}+k(1-2 a-2 \bar{n}) \chi^{4}\right], \\
B_{11}= & 2 G Y^{2}\left[(1+2 a) Z-2 k^{2}\left(4+5 \chi^{2}+\chi^{4}\right)\right], \\
B_{12}= & G Y\left\{8 G k ( 1 + \chi ^ { 2 } ) \left[2+4 a+(1+2 a+2 \bar{n}) \chi^{2}\right.\right. \\
& \left.+(a-\bar{n}-1) \chi^{4}\right]-G^{2} \chi^{2}\left[4+8 a+(6+4 a+4 \bar{n}) \chi^{2}\right.
\end{aligned}
$$

$$
\begin{aligned}
& \left.(1-2 a+2 \bar{n}) \chi^{4}\right]+k^{2}\left(4+5 \chi^{2}+\chi^{4}\right) \\
& \left.\times\left[4-2 a\left(4+2 \chi^{2}+\chi^{4}\right)+\chi^{2}\left(2+\chi^{2}+2 \bar{n}\left(\chi^{2}-2\right)\right)\right]\right\} \\
B_{21}= & 4 G Y^{2}\left[G^{2}(1+\bar{n})\left(-2+\chi^{2}\right) \chi^{2}-2 G k\left(1+\chi^{2}\right)\right. \\
& \left.\times\left((3+2 \bar{n}) \chi^{2}-4 n\right)-k^{2} \bar{n}\left(8+6 \chi^{2}-3 \chi^{4}-\chi^{6}\right)\right], \\
B_{22}= & G Y\left\{k ^ { 2 } ( 4 + 5 \chi ^ { 2 } + \chi ^ { 4 } ) \left[(1-2 a) \chi^{2}\left(4+\chi^{2}\right)^{2}\right.\right. \\
& \left.+2 \bar{n}\left(\chi^{2}-8\right)\right]-8 G k\left(1+\chi^{2}\right)\left[\bar{n}\left(\chi^{6}-8\right)-\chi^{2}\right. \\
& \left.\times\left(2+\chi^{2}-\chi^{4}+a\left(4+\chi^{2}\right)^{2}\right)\right]-G^{2} \chi^{2}[16-2 \bar{n} \\
& \left.\left.\times\left(\chi^{6}-8\right)+\chi^{2}\left(16+8 \chi^{2}-\chi^{4}+2 a\left(4+\chi^{2}\right)^{2}\right)\right]\right\}, \\
B_{31}= & G\left(2+3 \chi^{2}+\chi^{4}\right)\left\{k^{2}\left(4+5 \chi^{2}+\chi^{4}\right)[8 a+4 \bar{n}-4\right. \\
& \left.+(2 a-2 \bar{n}-1) \chi^{2}\right]+8 G k\left(1+\chi^{2}\right)\left[( 1 + \overline { n } ) \left(\chi^{2}\right.\right. \\
& \left.-2)-a\left(4+\chi^{2}\right)\right]+G^{2} \chi^{2}[4(2+2 a+\bar{n}) \\
& \left.\left.+\chi^{2}(2 a-2 \bar{n}-1)\right]\right\}, \\
B_{32}= & G Y\left\{-8 G k\left(1+\chi^{2}\right)\left[2+4 a-2 \bar{n}+(2+a+\bar{n}) \chi^{2}\right]\right. \\
& +G^{2} \chi^{2}\left[8 a-4 \bar{n}+(3+2 a+2 \bar{n}) \chi^{2}\right]+k^{2}\left(4+5 \chi^{2}+\chi^{4}\right) \\
& \left.\times\left[8 a-4(1+\bar{n})+(2 a+2 \bar{n}-1) \chi^{2}\right]\right\} \\
D_{11}=8 G Y Z\left(2+3 \chi^{2}+\chi^{4}\right)\left[2 G-k\left(4+\chi^{2}\right)\right], & Y^{2} \\
& D_{22}=\frac{\left(2+3 \chi^{2}+\chi^{4}\right)}{\left(D_{11},\right.}
\end{aligned}
$$

where $k$ is the cavity decay rate taken same for both the modes and

$$
\begin{gathered}
Y=\sqrt{\left(1+\chi^{2}\right)\left(-4+\chi^{4}\right)} \\
Z=G^{2} \chi^{2}-4 G k\left(1+\chi^{2}\right)+k^{2}\left(4+5 \chi^{2}+\chi^{4}\right) .
\end{gathered}
$$

[1] H. J. Kimble and D. F. Walls, J. Opt. Soc. Am. B 4, 1450 (1987).

[2] J. Hald, J. L. Sorensen, C. Sohori, and E. S. Polzik, Phys. Rev. Lett. 83, 1319 (1999); B. Julsgaard, A. Kozhekin, and E. S. Polzik, Nature (London) 413, 400 (2001).

[3] H. Xiong, M. O. Scully, and M. S. Zubairy, Phys. Rev. Lett. 94, 023601 (2005); H. T. Tan, S. Y. Zhu, and M. S. Zubairy, Phys. Rev. A 72, 022305 (2005).

[4] F. L. Li, H. Xiong, and M. S. Zubairy, Phys. Rev. A 72, 010303 (2005).

[5] Y. X. Ping, B. Zhanb, and Z. Cheng, Eur. Phys. J. D 44, 175 (2007).

[6] S. Qamar, M. Al Amri, and M. S. Zubairy, Phys. Rev. A 79, 013831 (2009); M. Al Amri, S. Qamar, S. Qamar, and M. S. Zubairy, Quantum Inf. Proc. 8, 587 (2009).

[7] S. Tesfa, Phys. Rev. A 79, 033810 (2009).

[8] J. Y. Li and X. M. Hu, Phys. Rev. A 80, 053829 (2009); X. Zhang and X. M. Hu, ibid. 81, 013811 (2010); F. Wang, X. M. Hu, W. X. Shi, and Y. Zhu, ibid. 81, 033836 (2010); W. X. Shi, X. M. Hu, J. Y. Li, and F. Wang, J. Phys. B 43, 155506 (2010).

[9] M. Ikram, G. X. Li, and M. S. Zubairy, Phys. Rev. A 76, 042317 (2007).

[10] S. Qamar, F. Ghafoor, M. Hillery, and M. S. Zubairy, Phys. Rev. A 77, 062308 (2008).

[11] X. M. Hu and J. H. Zou, Phys. Rev. A 78, 045801 (2008).
[12] S. Qamar, M. Al Amri, S. Qamar, and M. S. Zubairy, Phys. Rev. A 80, 033818 (2009).

[13] A. Fang, Y. Chen, F. Li, H. Li, and P. Zhang, Phys. Rev. A 81, 012323 (2010).

[14] V. Josse, A. Dantan, A. Bramati, M. Pinard, and E. Giacobino, Phys. Rev. Lett. 92, 123601 (2004).

[15] M. O. Scully, Phys. Rev. Lett. 55, 2802 (1985).

[16] M. O. Scully and M. S. Zubairy, Phys. Rev. A 35, 752 (1987); N. A. Ansari and M. S. Zubairy, ibid. 40, 5690 (1989).

[17] M. O. Scully and M. S. Zubairy, Quantum Optics (Cambridge University Press, Cambridge, UK, 1997).

[18] L. M. Duan, G. Giedke, J. I. Cirac, and P. Zoller, Phys. Rev. Lett. 84, 2722 (2000).

[19] R. Simon, Phys. Rev. Lett. 84, 2726 (2000).

[20] M. Hillery and M. S. Zubairy, Phys. Rev. Lett. 96, 050503 (2006).

[21] R. Tahira, M. Ikram, H. Nha, and M. S. Zubairy, Phys. Rev. A 79, 023816 (2009).

[22] G. Vidal and R. F. Werner, Phys. Rev. A 65, 032314 (2002).

[23] S. Sheel and D. G. Welsch, Phys. Rev. A 64, 063811 (2001).

[24] M. S. Kim, W. Son, V. Buzek, and P. L. Knight, Phys. Rev. A 65, 032323 (2002).

[25] W. Xiang-bin, Phys. Rev. A 66, 024303 (2002). 